\title{
VII.
}

Aus der inneren Abtheilung des Centraldiakonissenhauses Bethanien in Berlin (dirig. Arzt Sanitätsrath Dr. v. S teina u-Steinruck).

\section{Ein Beitrag zur Casuistik der Sublimatvergiftungen durch den inneren Gebrauch.}

\author{
Von \\ Dr. W. Frölich, \\ Assistenzarzt.
}

Mit der allgemeineren Einfuhbrung des Sublimats in die praktische Medicin mehrten sich auch die durch dieses Quecksilbersalz hervorgerufenen Vergiftungen. Die meisten derselben entstehen naturgemäss, der Art der Anwendung des Sublimats entsprechend, durch zu energischen äusserlichen oder auch subcutanen Gebrauch; und die Zahl der in der chirurgischen Praxis infolge von Behandlung grosser Wund. höhlen u.s.w. mit Sublimat auftretenden Intoxicationen bildet den weitaus grössten Procentsatz aller Sublimatrergiftungen. Dem gegenuber steht die versehwindend kleine Reihe der Sublimatvergiftungen nach dem inneren Gebrauch, meistens hervorgerufen durch Genuss dieses Giftes in selbstmörderischer Absicht. - Der Verlauf ist bei der innerlichen Vergiftung meistens bedeutend stirmischer und rapider als bei der äusseren, so dass eine Schilderung eines derartigen Krankheitsverlaufes von allgemeinem Interesse sein dürfte.

Im Folgenden theile ich 2 Fälle von acuter Sublimatvergiftung per os mit, die in den letzten Jahren im Centraldiakonissenhaus Bethanien-Berlin zur Beobachtung kamen.

Fall I. Betrifft die 29 Jahre alte Arbeiterin Id a S., sie giebt an, Vormittags um 1/211 Uhr 2 Sublimatpastillen, die ihr vom Arzte zu Sitzbädern verschrieben worden waren, mit Cacao zusammen genossen zu haben; es habe sich sofort Erbrechen eingestellt, und sie sei umgefallen.

Patientin, die Mittags zur Aufnahme kommt, macht den Eindruck einer schweren acuten Vergiftung, sie stöhnt fortwährend und findet sich im Zustande tiefsten Collapses. Radialpuls ist nicht zu fuhlen. Hände, Füsse, Nase und Ohren sind kalt; es besteht hohe Cyanose, Athemztige sind oberflächlich. An den Lippen, Mundwinkeln, Mundschleimhaut und Gaumen 
sind gelbliche Aetzschorfe. Magen und Leib druckempfindlich, keine Genitalblutung. Pupillen mittelweit, reagiren wenig; Zähne defect, Zahnfleisch aufgelockert, Zunge trocken; blutiger Stubl; Urin enthält reichlich Eiweiss. Herzthätigkeit stark beschleunigt, man hört nur einen Herzton. Lungen intact. Der Scheideneingang und Anfangstheil der Scheide ist schwarz, diphtherisch belegt, die Umgebung ist intensiv geröthet; sehr ubler Geruch.

Es erfolgen täglich zahlreiche diarrhöische Stühle, der Puls wird etwas kräftiger, am 3. Tage nach der Vergiftung tritt ganz plötzlich der Exitus ein. Wegen der gerichtlichen Beschlagnahmung der Leiche musste eine Section leider unterbleiben.

Fall II. Die Hebeamme Frau B., bisher immer gesund, hatte in einem Falle psychischer Erregung am 3. April Abends 7 Uhr 2 Sublimatpastillen (Angerer) in Cognac gelöst zu sich genommen. Bald darauf erfolgte heftiges Erbrecben. Nach 3 Stunden hatte sie auf Zureden ihres Mannes ca. 2 Liter Milch getrunken; gleichzeitig erfolgten fortwährend diinne, wässrige, nicht blutige Stuhlentleerungen, welche bis zum nächsten Morgen anhielten. Am Tage darauf war das Allgemeinbefinden besser, die Stuhlgänge wurden seltener und erfolgten jetzt etwa stindlich, ebenfalls obne Blut oder Schleimhautfetzen. Urin wurde normal entleert: es stellten sich heftige brennende Schmerzen in Magen und Darm ein. Therapie: Milch und Eiweiss.

Am 5. April trat völlige Anurie anf, die Durchfälle hörten auf, Erbrechen war selten. Milch wurde gut vertragen. Sensorium war frei, Patientin klagte über Athembeschwerden und Rlickenschmerzen.

Am 6. April stellte sich Oedem des Gesichtes ein, der Puls war in der ganzen Zeit gut, 80-100 in der Minute; Temperaturen stiegen nicht tiber $38^{\circ}$. An den drei folgenden Tagen wurden Schwitzkuren versucht. Das erste Mal mit einem guten Erfolge, so dass Patientin aufstand und Zeitungen las. Die Anurie bestand bis zum 10. April fort; im Allgemeinen blieb der Zustand derselbe.

Am 11. April, dem Tage der Einlieferung in das Krankenhaus, ergab sich folgender Status: Frau, kräftig gebaut, starke Adipositas, Gesicht etwas gedunsen, leichte Oedeme, keine Sehstörung, Reflexe normal, Sensorium frei. Zunge stark belegt, trocken, rissig, auf der Wangenschleimhaut oberflächliche, leicht blutende Ulcerationen. Die Tonsillen sind geschwollen, auf denselben ebenfalls Ulcerationen. Häufig Erbrechen dünner, grünlich gefärbter, gallig schmeckender Massen ohne Blut. Abdomen ist weich, nicht schmerzhaft, keine Dämpfung. Anurie. Seit 4 'Tagen ist Patientin menstruirt, Blutung mässig. Herz: Grenzen nicht erweitert, leises systolisches und diastolisches Geräusch; Puls voll 96. Lungen: Respiration 42, keine Dämpfung, auf beiden Lungen raubes Athmen, Rhouchi; - Temp. $36,8^{\circ}, 36,9^{\circ}$.

Ordinirt wurde: Eisstilckchen, Milch, Buttermilch.

Am 11. April Abends wird vermittelst Katheter ca. $50 \mathrm{Cm}$. Urin entleert; derselbe ist gelb, trübe; Albuminurie: 4 Proc., er enthält zahlreiche Epithelcylinder und homogene Cylinder, sehr wenige rothe Blutkörperchen. Das Erbrechen bat sehr zugenommen, es erfolgt fast bei 
jeder Bewegung. Schlaf stelit sich nacb einer Morphiuminjection von 0,005 ein. $36,2^{0}$.

Am 12. April. Status idem. Urin: $100 \mathrm{Ccm}$. (Katheter); Temp. $36,2^{0}$,

Am 13. April. Erbrechen steigert sich. Urin $350 \mathrm{Ccm}$; Schwitzkur ohne Erfolg.

Am 14. April. Grenzen des Herzens deutlich erweitert, bei Systole und Diastole lautes, scharfes, kratzendes Geräusch (Pericarditis sicca). Lungen: unten binten links Dämpfung, scharfes, bronchiales Athmen. Magengegend druckempfindlich; Abdomen zeigt Dämpfung in den abhängigen Theilen. 1 Stuhl, dtinn, kein Blut, Urin $365 \mathrm{Ccm}$.

15. April. Das kratzende Geräusch am Herzen fast verschwunden; Herzdämpfung stark vergrössert. Töne leise, kaum hörbar, Spitzenstoss innerhalb der Dämpfungsfigur. Temp. 36,00, 35,70 Urinmenge $360 \mathrm{Ccm}$; Sensorium zeitweise etwas benommen.

16. A pril. Zunehmender Stupor. Pat. versteht schwer, merkt nicht, dass man mit ihr spricht. Gegen Abend heftige Schmerzen in der Brust beim Athmen; scharfes, pleuritisches Reiben besonders rechts vorn. Auf beiden Lungen starkes Rasseln.

17. April. Pat. schreit die ganze Nacht durch; morgens 9 Uhr erfolgt unter einzelnen Convulsionen der Exitus.

Sectionsbefund: Starker Paniculus adiposus. Im Herzbeutel Ansammlung einer grossen Menge seröser Flüssigkeit; das Herz ist in toto vergrössert; die Aussenfläche desselben mit Fibrinfasern bedeckt (cor villosum) Wandungen sind verdickt, zeigen fettige Degeneration. Lungen: An der Aussenfläche der rechten Lunge sind frische pleuritische Aufagerungen. Lunge selbst lufthaltig, in den Bronchien schaumige Flussigkeit. Pleura der linken Lunge zeigt ebenfalls fibrinöse Auflagerung; im Pleuraraum eine mässige Menge seröser Flïssigkeit; linker Unterlappen fuhlt sich fest an, in den Bronchien schaumige Flüssigkeit. Lar y nx: Injicirte Schleimhaut; leichtes Oedem der Glottis, stärkeres Oedem der Epiglottis und der Aryknorpel. Mage n zeigt das Bild einer acuten Gastritis; Schleimhaut aufgelockert, Schleimhautfalten stark geröthet, ebenso zeigt die Schleimbaut des Darmes acute Reizung, und zwar im Dünndarm in der Nähe des Coecum und besonders im Dickdarm am Coecum starke Röthung und Schwellung der Schleimhaut, auf der Höhe der Schleimhautfalten zahlreiche Ekchymosen. Milz nicht besonders vergrössert, auffallend hart. Le b e r vergrössert, Acinizeichnung verwischt; Fettinfiltration. Ni e r e n stark vergrössert, Gewicht je $360 \mathrm{Grm}$; Oberfläche glatt, von fast weisser Farbe, Rindensubstanz verbreitert; von gelblicher Färbung. Die mikroskopische Untersuchung ergiebt ausgedehnte fettige Degeneration der Rindensubstanz.

Es mögen nun noch 10 Fälle von acuter Sublimatvergiftung durch Verschlucken folgen, die aus den Jahresberichten von Virchow und Hirseh der letzten 25 Jahre zusammengestellt sind.

Fall 1. (Philad. med. and. surg. Report. 72.)

Pat. nahm eine Unze concentrirter alkoholischer Sublimatlösung zu sich. Sofort stellte sich Erbrechen von Blut, später auch von Schleim- 
hautfetzen aus dem Magen ein; daneben bestanden profuse Durchfälle von ähnlicher Beschaffenheit. Gleich Anfangs trat ein intensiver Collaps ein. Allmählich liessen die Erscheinungen nach; Heilung.

Fall 2. (Woodbury, Philad. med. Times. 72.)

Frau, nahm 4,0 alkoholische Sublimatlösung zu sich; gleich darauf starkes Bluterbrechen; nach 3 Stunden wurden als Gegenmittel rohe Eier gereicht. 2 Tage lang bestand Erbrechen, blutige Diarrhoe, Uebelkeit, metallischer Geschmack, Salivation. Dann erfolgte zunehmender Collaps; am 8. Tage Exitus letalis.

Bei der Section fand sich die Magenschleimhaut und zum Theil auch das Duodenum in einem entzindlichen, erweichten Zustande; durch extravasirtes Blut war das Aussehen derselben missfarben geworden.

Fall 3. (0livier, Archiv de physiol. norm. et patholog. 73.)

Fran, 62 Jahre alt, nahm 1,12 Sublimatlösung zu sich; es wurde ein augenblickliches Sinken der Temperatur von $37,7^{\circ}$ auf $36,8^{\circ}$ beobachtet. Es bestand Erbrechen und profuse Durchfälle. Der Urin zeigte vom 2. Tage ab Albumen, dessen Menge allmählich abnahm, mikroskopisch fand man zahlreiches, fettig degenerirtes Nierenepithel und gekörnte Cylinder. Exitus erfolgte am 6. Tage.

Fall 4. (Oettinger, Leistung und Fortschritte der gesammten Medic. 1876.)

Knabe, anämisch, luetisch; die genommene Dosis enthielt 0,70 Sublimat. Gleich darauf acute Gastro-Enteritis, plötzlicher Temperaturabfall, Haematemesis, Coma. Am 2. Tage ausser den genannten Erscheinungen Retentio urinae, Trismus, blutige Stuhle. Am 4. Tage schleimige Entleerungen, Stomatitis, Glossitis. Krämpfe in den unteren Extremitäten, links mit folgender Parese; Bronchitis. Mit der 2. Woche ausgesprochene Symptome des chronischen Mercurialismus: Haut braun und trocken, später Erythem, Ekzem, Pusteln. Salivation aussergewöhnlich stark bis za $8000,0 \mathrm{Ccm}$.! täglich. Uleera im Munde, Dysphagie, Cardialgie, Ikterus catarrhalis, Cystitis, Oedem; Temp. 40,80, starke Schweisse. Der Urin enthielt 3 Monate lang Albumen und Quecksilber; dann traten Lockerung und Ausfallen der Zähne ein, ferner Abstossen nekrotischer Knochenstücke des Kiefers und endlich noch Chorea minor. - Heilung nach 2 Jahren.

Fall 5. (M a chka, Vierjahrsschrift für Heilkunde, Prag.)

Mädchen, erwachsen, nahm 250,0 Grm. einer Sublimatlösung zu sich, deren Stärke nicht zu bestimmen war; starkes Glottisödem; Exitus nach kurzer Zeit. Section: Im Magen ein Lalber Liter chocoladenfarbiger Flussigkeit, neutral reagirend, Schleimhaut stark geschwollen, gelblich grau. Inhalt des Dunndarmes blutig gefärbt, Schleimhaut dunkelroth, geschwollen, mit zahIreichen Ekchymosen versehen. Starke Schwellung der Follikel und Peyer'schen Plaques. In der Blase blutig gefärbter Urin.

Fall 6. (Barthellung, Annales d'hyg. publ.)

Mann, kräftig, nimmt $70-80 \mathrm{Ccm}$. concentriter Sublimatlösung zu sich. Unmittelbar darauf Gastro-Enteritis, graue Verschorfung der Mundschleimhaut, später heftige Stomatitis, Salivation. Vom 5. Tage ab starke Albuminurie; Exitus am 9. Tage unter plötzlichem Collaps.

Section: Im Schlingtractus bereits gelockerte Schorfe, starke Injection, 
starke Schwellung der Magenschleimhaut, des Duodenum, besonders des oberen Theiles des Jejunum und des Dickdarmes, hier zahlreiche Ekchymosen. Ileum normal. Fettige Degeneration der Nieren; in Herz und Leber keine fettige Entartung nachweisbar.

Fall 7. (M a schka, Prager medicin. Wochenschrift 1883.)

36 Jahre alte Frau, nimmt eine grössere Menge concentrirte Sublimatlösung zu sich: sofort heftiges Erbrechen, dyssenterische Sttihle, heftige Schmerzen im Leibe. Exitus nach 6 Stunden.

Section: Schleimhaut der Lippen, des Mundes, der Zunge grau gefärbt, gerunzelt, wie gegerbt, Epithel abgelöst, Schleimhaut der Speiseröhre ähnlich aussehend, im Magen mässige Menge eines blutig gefärbten, mit Schleim untermenıten breiigen Inhaltes; Schleimhaut hämorrhagisch, nekrotisch, gewulstet, in eine braunröthliche, schmierige Masse verwandelt. Magenwandung tiefverändert, Gefässe mit Gerinnseln angefüllt. Im Darmkanal flussiger, blutig gefärbter Inhalt, Schleimhant des Dünndarmes blutig suffundirt, in den Dickdarm hinein allmählich geringer werdend. Milz, Leber normal. Nieren mittelgross, Kapsel leicht ablösbar, Gewebe dunkelroth, an der Basis der Pyramiden kleine Ekchymosen.

Fall 8. u. 9. (Maschka, Prager medicin. Wochenschrift 1884.)

Ein junges Paar nahm eine nicht $z u$ bestimmende Menge Sublimatlösung $\mathrm{zu}$ sich.

a) Beim Mädchen, 28 Jahre alt, gleich nach dem Genusse heftiges Wïrgen und Erbrechen, herber Geschmack im Munde, brennender Schmerz im Schlunde und Magen. Schleimhaut des Mundes normal. Puls klein, 90, Temp. normal; starker Durst, Singultus. Tags darauf: Status im Allgemeinen derselbe, drei gewöhnliche Stuble, im Urin viel Albumen. im Laufe des nächsten (also 3.) Tages sieben diarrhoeische, darauf elf blutige Stühle, Schmerzen links im Unterleibe.

Am 4. Tage: sieben blutige Stuhle, Erbrechen grünlicher Massen, heftiger Durst, Anurie, Temp. subnormal.

Am 6. Tage: Erbrechen und Diarrhoe geringer; starke Stomatitis; Anurie besteht fort.

Am 7. Tage: starker Collaps; unter Convulsionen erfolgt Exitus.

Section: Herz normale Grösse, Musculatur dunkelroth, keine fettige Degeneration. Lungen: frei, Unterlappen blutreich, Oberlappen ödematös. Schleimhaut des Oesophagus im unteren Drittel bräunlich verfärbt, Epithel theils abgelöst in Fetzen herabhängend. Leber: Grösse normal, dunkelbraun, blutreich, geringe fettige Infiltration. Nieren: vergrössert, Kapsel leicht ablösbar, Oberfläche glatt, Rindensubstanz verbreitert, blass, Verfettung des Epithels der Harnkanälchen. Ma g en: Schleimhaut leicht geschwollen, am Grunde etwas geröthet, einzelne Ekchymosen. $\mathrm{Sch}$ le i m h a t des Dickdarmes vom Mastdarm bis zum Coecum geschwollen, dunkelbraunroth, mit gelbgrunen, symmetrisch angeordneten Schorfen bedeckt; an einzelnen Stellen Schorfe abgelöst; tiefgehende Substanzverluste ohne Perforation. An der Schleimhaut des Dinndarmes nur bei der Cöcalklappe Verschorfung, Injection und Auflockerung.

b) Mann, 25 Jahre alt, bald nach dem Genusse des Sublimats hochgradige Angst, theilweise grauweisse Verfärbung der Schleimhant des 
Mundes, Brennen im Munde, Oesophagus, Magen und Unterleib, heftiger Durst, fortwährendes Erbrechen, continuirlicher Stuhlgang, dünne, scbleimige Stible.

Am nächsten Tage mässiger Tremor der Extremitäten, schaumige, blutige Stuhle.

Am 4. Tage: Nachlassen der Erscheinungen, sonst Status idem.

Am 5. Tage: auffallende Euphorie $3 / 4$ Stunde vor dem Tode, Collaps, Exitus letalis.

Section: Herz: Herzbeutel normal. Lungen: frei, in den Bronchien schleimige Flüssigkeit, in der linken Pleurahöhle geringes, blutiges Exsudat. Zunge: bräunlich belegt, Schleimhaut wenig verändert, Schleimhaut der Trachea geröthet, schaumiger Inhalt. Speiseröhre: bräunlich verfärbt, leicht gerunzelt, Epithel abgelöst. Bauchhöhle: in derselben mässige Menge einer trüben, blutigen Flüssigkeit, Netzgefảsse stark injicirt. Magenwandung von aussen bräunlich verfärbt, derb, fest, im Magen eine bräunliche, neutrale Flussigkeit. Schleimhaut dunkelbraun, gewellt, blutig suffundirt, tief verschorft, Epithel abgelöst. D u n nd a r m: etwas gewellt, stellenweise injicirt. D i c k d a r m : hochgradig verschorft, blutig suffundirt. Ni e r e n: vergrössert, Kapsel leicht ablösbar, Rindensubstanz verbreitert, Pyramiden blass, hochgradige Verfettung. M il z: vergrössert, blutreich. Le ber: normale Grösse, Oberfläche glatt; mässige Fettinfiltration.

Fall 10. (Bä umler, Zeitschrift flur medicinische Beamte.)

Junger Mann, Stud., nimmt irrthümlicher Weise statt Antipyrin 4,5 Grm. Sublimat. Sofort heftiges Erbrechen von Blut, zahlreiche dyssenterische Stahle, ebenso trat sofort Auurie auf. Vom 2. Tage an bestand auffal lende Euphorie, am 5. Tage piötzlich Auftreten urämischer Erscheinungen, die bis zum bald darauf erfolgenden Exitus anhielten.

Section: Magen und grösster Theil des Dünndarmes von normaler Farbe. Im Magen, kurz vor dem Pylorus, ein 5 Qucm. grosser, weicher Schorf, unter demselben ödematöse Schwellung des submukösen Bindegewebes. Serosa unverändert. Schleimhautfalten, die vom Schorf zur Cardia ziehen, sind hellgelblich verfärbt. Oesophagus und cardialer Theil des Magens zeigt etwas Injection. Unterer Theil des Ileum, des Coecum, Colon bis Rectum zum Theil gangränös. Die Nieren auffallend gross, starke parenchymatöse Schwellung.

Von den angefuihrten Fällen sind nur zwei zur Heilung gelangt; es ergiebt sich mithin eine Mortalität von 82 Proc. Fassen wir noch einmal die Symptome zusammen, die sicb bei den eben geschilderten Fällen immer wiederholen, so sind dies: mehr oder weniger hervortretender metallischer Geschmack, Schwellung und Ulceration der Schleimbaut des Mundes und Rachens, Brennen im Schlunde, Schmerzen im Leibe, Würgen, Erbrechen diunnflüssiger, häufig blutig gefärbter Mengen, profuse oft blutige Stühle, Tenesmus, geringe, stark eiweisshaltige Urinmenge oder gar Anurie. - Auffallend ist in einigen Fällen die oft bis zum Tode andauernde Euphorie, die sich nicht einmal durch die schliesslich auftretendeu Convulsionen beeinflussen lässt. 
Ein weiteres Moment, das wohl Beachtung verdient, findet sich bei dem zweiten in Bethanien beobachteten Falle in der aussergewöhnlich sttirmischen Betheiligung des Herzens und des Herzbeutels. Während wir bei den übrigen Fällen entweder keine oder nur ganz geringe Betheiligung von Seiten des Herzens gesehen haben, lässt sich hier eine schwere acute Entzïndung des Herzens und Herzbeutels mit allen ihren Folgeerscheinungen constatiren.

Eine auffallende Erscheinung, welche noch der Aufklärung bedarf, ist die in Betreff des Darmtractus. Bei allen Vergiftungen durch Sublimat, welche zur Section kamen, zeigte sich im Darme die grösste Veränderung stets im Dickdarm, und zwar in der Nähe des Coecums, und in dem diesem Theile angrenzenden Dünndarm. Das Duodenum und der grössere Theil des Dünndarmes waren in höchst seltenen Fällen an den Veränderungen betheiligt. - Wie gesagt, eine befriedigende Erklärung für diesen Vorgang giebt es noch nicht. Auch die Ansicht, dass die im Dickdarm aufgestauten Fäcalmassen hierselbst (das Sublimat) eine längere und directe ätzende Einwirkung des Sublimats beguinstigen, ist doch auch sehr wenig bewiesen, besonders nachdem durch experimentelle Versuche an Thieren dargethan ist, dass die Sublimatwirkung auch dann an diesen Stellen zum Vorschein kommt, wenn das Gift subcutan applicirt wurde. 